\title{
Ultra-high precision $x$-ray polarimetry with artificial diamond channel cuts at the beam divergence limit
}

\author{
Hendrik Bernhardt, ${ }^{1,2}$ Annika T. Schmitt, ${ }^{1,2}$ Benjamin Grabiger $\odot,{ }^{1,2}$ Berit Marx-Glowna, ${ }^{1,2}$ Robert Loetzsch,,${ }^{1,2}$ \\ Hans-Christian Wille $\odot,{ }^{3}$ Dimitrios Bessas $\odot,{ }^{4}$ Aleksandr I. Chumakov $\odot,{ }^{4}$ Rudolf Rüffer, ${ }^{4}$ Ralf Röhlsberger, $, 1,2,3$ \\ Thomas Stöhlker, ${ }^{1,2}$ Ingo Uschmann,,${ }^{1,2}$ Gerhard G. Paulus $\circledast,^{1,2}$ and Kai S. Schulze $e^{1,2, *}$ \\ ${ }^{1}$ Institut für Optik und Quantenelektronik, Friedrich-Schiller-Universität Jena, Max-Wien-Platz, 1, 07743 Jena, Germany \\ ${ }^{2}$ Helmholtz-Institut Jena, Fröbelstieg 3, 07743 Jena, Germany \\ ${ }^{3}$ Deutsches Elektronen-Synchrotron DESY, Notkestraße 85, 22607 Hamburg, Germany \\ ${ }^{4}$ ESRF-The European Synchrotron, CS 40220, 38043 Grenoble Cedex 9, France
}

(Received 3 December 2019; accepted 28 May 2020; published 19 June 2020)

\begin{abstract}
We report on the use of synthetic single-crystal diamonds for high definition x-ray polarimetry. The diamonds are precision mounted to form artificial channel-cut crystals (ACCs). Each ACC supports four consecutive reflections with a scattering angle $2 \Theta_{\mathrm{B}}$ of $90^{\circ}$. We achieved a polarization purity of $3.0 \times 10^{-10}$ at beamline ID18 of the European Synchrotron Radiation Facility (ESRF). When the x-ray beam's horizontal divergence was reduced through additional collimation from 17 to $8.4 \mu \mathrm{rad}$, the polarization purity improved to $1.4 \times 10^{-10}$. Precision $\mathrm{x}$-ray polarimetry thus has reached the limit, where the purity is determined by the divergence of the beam. In particular, this result is important for polarimetry at fourth generation x-ray sources, which provide diffraction-limited x-ray beams. The sensitivity expected as a consequence of the present work will pave the way for exploring new physics such as the investigation of vacuum birefringence.
\end{abstract}

DOI: 10.1103/PhysRevResearch.2.023365

\section{INTRODUCTION}

The discovery of the polarization of $\mathrm{x}$ rays by Barkla in 1904 [1] was decisive for their identification as electromagnetic waves. Today, polarized $\mathrm{x}$ rays enable a large array of applications, spurred by the advent of modern synchrotron x-ray sources, which, in turn, have also fostered the development of x-ray polarizers and polarimeters [2-7]. The extinction ratio of the latest $\mathrm{x}$-ray polarimeters has reached the level of $10^{-10}$ [6], which is unique in the entire field of optics. As a consequence, a number of new scientific opportunities has emerged in different fields of $x$-ray science. Examples include nuclear resonant scattering [2-4] and x-ray quantum optics [8-10]. There, precision x-ray polarimeters efficiently discriminate the huge number of photons of a synchrotron against a handful of scattered photons. Other applications are under intense development at, e.g., the European X-ray Free Electron Laser. One example is ultrafast imaging of the transient ultrastrong magnetic fields emerging in laser-driven solid-density plasmas via the Faraday effect [11]. Quite generally, the sensitivity increases linearly with the polarization purity, defined as the intensity ratio of undesired to desired polarization state [2]. Further improvements are therefore of great interest.

\footnotetext{
*kai.sven.schulze@uni-jena.de

Published by the American Physical Society under the terms of the Creative Commons Attribution 4.0 International license. Further distribution of this work must maintain attribution to the author(s) and the published article's title, journal citation, and DOI.
}

A particularly demanding application of x-ray polarimeters, which actually sets the benchmark for our efforts, is the detection of the birefringence of vacuum polarized by an extremely strong laser field [12-14]. Based on the ideas of Euler and Heisenberg [15], the effect was already predicted in the early days of QED [16]. Like in birefringent crystals, the induced optical anisotropy causes a difference between the refractive indices parallel and perpendicular to the electric or magnetic field vector. As a consequence, a linearly polarized probe beam is converted into an elliptically polarized one upon propagation through the highly intense field. The induced ellipticity $\delta$ scales linearly with the photon energy. Experimentally, only intensities can be measured. Thus, the quantity to determine is $\delta^{2}$, which, accordingly, scales with the square of the photon energy. Therefore, it is expedient to use $\mathrm{x}$ rays as a probe. Estimates predict that $\delta^{2}$ is in the order of $10^{-12}$ for $10-\mathrm{keV}$ photons and a multi100-TW laser [12]. In order to make vacuum birefringence detectable, it is the quest to realize polarization purities of $10^{-11}$ or better, to develop precision polarimeters for higher photon energies, and to get the highest possible laser fields. Possible facilities, where intense laser and x-ray beams are available, or will be available in the near future, are the MEC instrument at the Linac Coherent Light Source in California, the HED instrument at the European XFEL in Germany, the Shanghai Coherent Light Facility in China, and BL2 of the SPring-8 Angstrom Compact Free-Electron Laser in Japan. An intriguing aspect of vacuum birefringence experiments is the potential to discover hypothetical elementary particles beyond the standard model $[17,18]$.

Our polarimeters rely on a series of Bragg reflections with a scattering angle $2 \Theta_{\mathrm{B}}$ of $90^{\circ}$, where $\Theta_{\mathrm{B}}$ is the Bragg angle. 


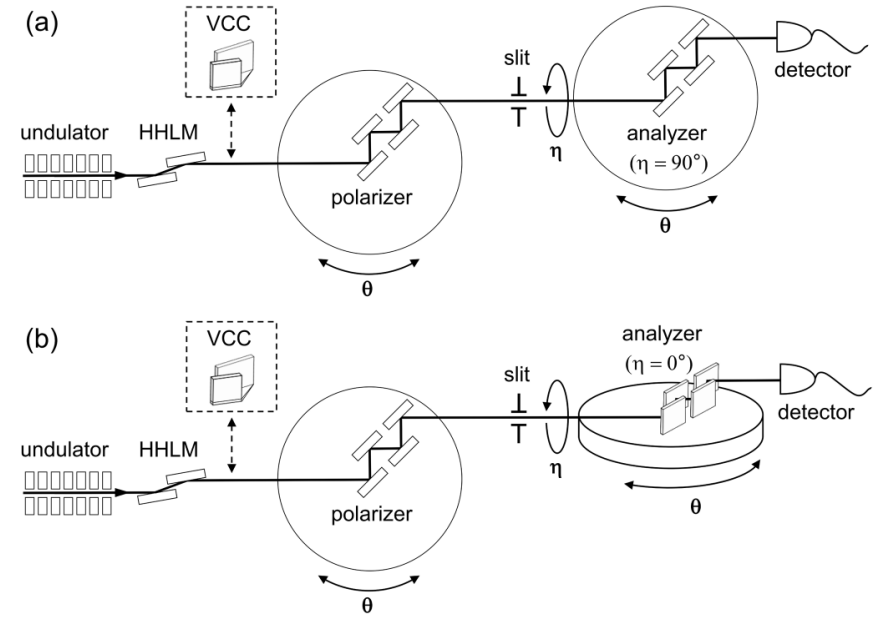

FIG. 1. Scheme of the experimental setup with the analyzer (a) in parallel and (b) in crossed position. The high-heat-load monochromator (HHLM) narrowed the spectral bandwidth of the x-ray beam. An inserted V-shaped channel cut (VCC) or a slit reduced the horizontal beam divergence as required.

The easiest way to realize this is the reflections at the walls of a channel cut into a crystal. We already demonstrated a purity of $2.4 \times 10^{-10}$ at a photon energy of $6.45 \mathrm{keV}$ using specially designed monolithic silicon crystals which support six consecutive $90^{\circ}$ reflections [6]. Due to its thermal properties, but also due to its smaller lattice constant and lower atomic number, which help in avoiding detrimental multiple beam reflections, diamond is a particularly attractive material for $\mathrm{x}$ ray polarizers. Recently, we showed that polarizers consisting of only two precisely aligned diamond crystals enable a purity of $8.9 \times 10^{-10}$ at $9.839 \mathrm{keV}$ [7].

However, it has not been possible to surpass the record figure already achieved in 2013, i.e., a polarization of $2.4 \times$ $10^{-10}$. In this paper, by manipulating the divergence of the $\mathrm{x}$-ray beam, we present experimental evidence that the polarization purity is limited by the divergence of the x-ray beam as predicted in a recent theoretical work [19]. In addition, we demonstrate polarizers consisting of four individually precision-controlled diamond crystals and report a record of $1.4 \times 10^{-10}$ at $9.839 \mathrm{keV}$. More importantly, this work implies plenty of room at the bottom for $\mathrm{x}$-ray polarizers in consideration of the order-of-magnitude lower divergence of $\mathrm{x}$-ray free-electron lasers.

\section{FACTORS AFFECTING THE POLARIZATION PURITY}

A general polarimetric setup consists of two polarizers. An example is shown in Fig. 1, which is a sketch of our experimental setup. The first polarizer selects a certain polarization state out of the primary beam. In the case of synchrotron setups, the polarizer enhances the degree of linear polarization of the undulator radiation. The second polarizer, called the analyzer, selects a certain polarization state as well and can, therefore, analyze the created polarization when rotated around the beam. In the parallel setting, where the scattering planes of the polarizer and analyzer are parallel, both transmit the same polarization state, whereas in the crossed setting the polarization states are orthogonal and the overall transmission of the polarimeter is minimum. Polarizers and polarimeters in the x-ray range have been used for decades [20-22] and more recently with the highest sensitivity [5-7].

In order to reach the ultimate sensitivity of an instrument, the influence of every component has to be investigated. In the case of x-ray polarizers, deviations of the scattering angle $2 \Theta_{\mathrm{B}}$ from $90^{\circ}$ are an obvious limitation, since the polarization component parallel to the scattering plane is different from zero. As a consequence of x-ray diffraction, the incident angle or rather the Bragg angle is connected with the photon energy by the Bragg condition. In order to reach the highest polarization purities, this demands an accurate energy alignment. For two reflections at diamond, the deviation has to be smaller than $\pm 0.5 \mathrm{eV}$ in photon energy or $\pm 0.003^{\circ}$ in Bragg angle to reach purities in the order of $10^{-10}$. Increasing the number of reflections to four relaxes the requirements to $\pm 5 \mathrm{eV}$ or $\pm 0.03^{\circ}$ and, hence, makes the setup more robust to the alignment state. These values assume perfect crystals and are computed according to the dynamical theory of $\mathrm{x}$ ray diffraction. The requirements are less harsh for distorted crystals.

As already reported in Ref. [6], the reachable polarization purity can also be limited by multiple-wave diffraction, where the beam is diffracted at additional lattice planes. Even if the Bragg condition is not fulfilled for these planes, the long tails of the reflection curves will diffract radiation in the direction of the ongoing beam path via a detour (called Umweganregung by Renninger [23]). The unwanted polarization is no longer suppressed, effectively resulting in an elliptically polarized beam. The number of contributing lattice planes increases with photon energy. For this reason, higher harmonics of the synchrotron radiation have to be suppressed as much as possible. Furthermore, multiple-wave diffraction has to be reduced by a careful alignment of tilt and yaw of the diffraction planes.

Moreover, the choice of an appropriate crystal material is imperative to reduce multiple-wave diffraction. The influence of multiple-wave diffraction grows with the fourth power of the atomic number $Z$ [24]. Silicon with $Z=14$ is advantageous because perfect crystals with minimal impurities are readily obtainable and can easily be machined. For the challenge of detecting the birefringence of vacuum, the highest polarization purity at the highest photon energy is desired. Obviously, diamond with $Z=6$ is an attractive material. For example, the 004 and 008 Bragg reflection go along with a photon energy of 9.83 and $19.66 \mathrm{keV}$, respectively.

For highly symmetrical structures like the diamond cubic one, there are only a few allowed Bragg reflections, which can contribute to multiple-wave diffraction. If the crystal is aligned to a highly symmetrical point, all polarization changing wave fields interfere destructively and extreme purities are achievable [19]. In the case of the diamond crystal structure, the 004 Bragg reflection provides such a symmetry, if the projection of the incident beam onto the lattice planes is parallel to the $\langle 110\rangle$ direction. In order to reach polarization purities of $10^{-11}$, the deviation from this direction has to be smaller than $\pm 2.5^{\circ}$. The crystals were prealigned accordingly. During the experiment, the azimuthal angle of the analyzer 
crystal was varied by $\pm 1^{\circ}$ to verify the negligible influence of multiple-wave diffraction.

An $\mathrm{x}$-ray polarimeter using diamonds benefits also from further properties. First, there is low thermal diffuse scattering because of a small Debye-Waller factor. Second, a peak reflectivity of up to $100 \%$ is possible for nearly perfect crystals [25].

There are also challenges when dealing with diamond such as price, availability, size, and quality. Contrary to silicon, available diamonds contain a significant amount of dislocations and stacking faults. Nevertheless, we were able to realize artificial diamond channel cuts recently. For this, we developed a crystal holder that allows us to align two small crystals very precisely parallel to each other. In 2016, we used two of these channel cuts as a polarizer and analyzer for a polarimeter setup and achieved instantly a polarization purity of $8.9 \times 10^{-10}$ at $E_{\mathrm{ph}}=9.83 \mathrm{keV}$ [7].

Therefore, we continued to develop a diamond channel cut with four crystal reflections in order to explore the limitations beyond the $10^{-9}$ purity level.

In a theoretical analysis [19], the divergence was identified as one of such limitations. The limit of the polarization purity $P_{\text {limit }}$ can be estimated by the formula

$$
P_{\text {limit }}=\sigma_{\text {ver }}^{2}+\sigma_{\text {hor }}^{2},
$$

where $\sigma_{\mathrm{ver}}$ and $\sigma_{\mathrm{hor}}$ are the root-mean-square (rms) divergences in the vertical and horizontal direction, respectively. It is important to note that $P_{\text {limit }}$ cannot be improved by a higher number of reflections inside the channel cuts. For polarization purities of less than $10^{-10}$, rms beam divergences of $7 \mu \mathrm{rad}$ or less are required. The horizontal beam divergence of a third generation synchrotron source such as APS, ESRF, PETRA III, and SPring-8 is usually in the order of $10 \mu \mathrm{rad}$, limiting the purity according to Eq. (1) to $2 \times 10^{-10}$. To achieve better polarization purities, the divergence has to be reduced.

\section{DIAMOND POLARIZERS}

In order to demonstrate that we can reach a limit where the polarization purity is determined by the divergence of the beam, we developed two artificial channel cuts (ACCs) each holding four diamond crystals. A model of the ACC is shown in Fig. 2. It contains four holders for $8 \times 8 \times 1.2 \mathrm{~mm}^{3}$ single-crystal diamonds. The first crystal is fixed, whereas the second to fourth can be aligned in pitch and tilt by mirror mounts, which are common in laser optics but used as crystal mounts here. For a stable operation, this alignment is realized by piezo inertia actuators. The mirror mounts visible on the left side of Fig. 2 are nested into each other in order to get a compact and stable setup. An important feature of the ACC is a photodiode on a translation stage which can be moved through the channel. This allows the separate measurement of intensity behind the first, second, and third crystal. Together with a detector behind the ACC, all crystals can be aligned precisely parallel to each other. Both ACCs are mounted on goniometers to adjust the incidence angle close to $45^{\circ}$.

Crucial for optimum polarization purity is the photon energy $E_{\mathrm{ph}}$. It should be chosen in such a way that the Bragg angle $\Theta_{B}$ is exactly $45^{\circ}$ for the used Bragg reflection (here diamond 004). Note that the incident angle, measured to the

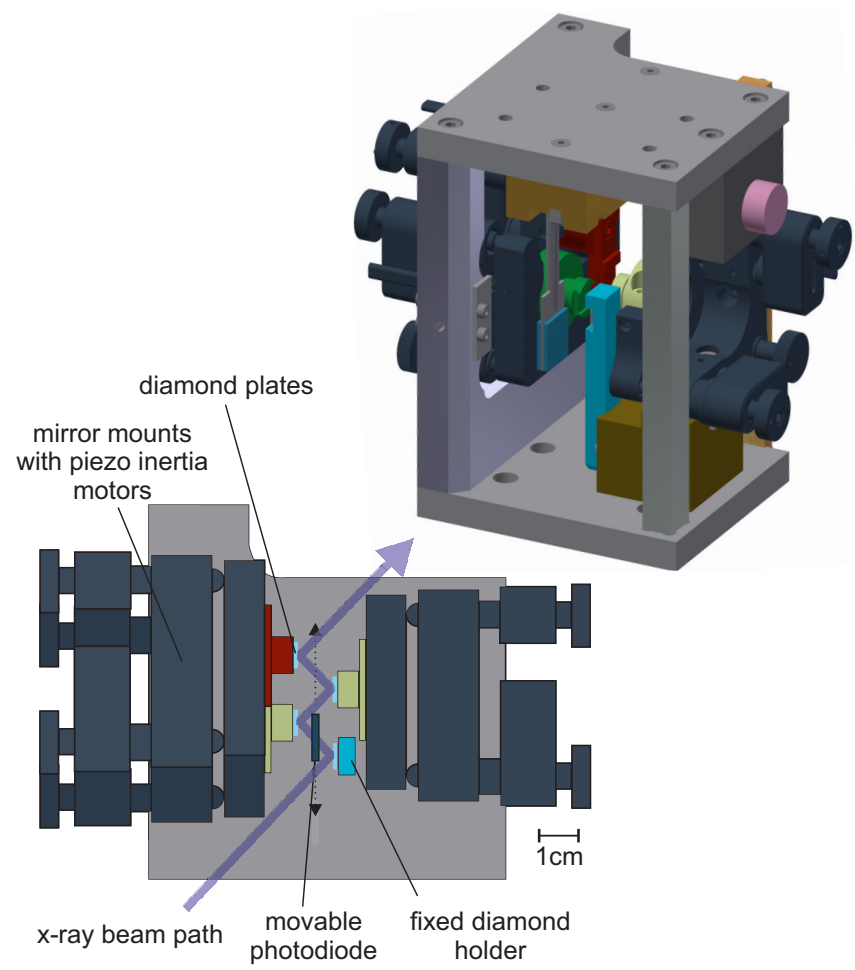

FIG. 2. Schematic view of the compact artificial channel cut in three dimensions and in top view. Pitch and tilt of three of the four diamond crystal plates can be aligned using motorized mirror mounts in order to arrange the crystals as parallel as possible. These mounts are fixed to a cage made of Invar steel.

lattice planes, has to be $15 \mu \mathrm{rad}\left(0.0009^{\circ}\right)$ larger than the Bragg angle of $45^{\circ}$ because of refraction at the crystal surface. This deviation is much less than the required $0.03^{\circ}$ accuracy and can therefore be neglected. For a given Bragg angle, $E_{\mathrm{ph}}$ is linked via the Bragg equation to the lattice constant $a$. The literature value of $a$ ranges from 3.56682 to $3.56714 \AA$. It depends on temperature, the fraction of ${ }^{13} \mathrm{C}$ isotopes $[26,27]$, the concentration of nitrogen and boron impurities [28,29], and also on the amount and type of crystal defects [30]. In our experiment, we used artificial diamonds produced by chemical vapor deposition (CVD). For these crystals, we measured $a=3.56698(8) \AA$ using the method of Bond $[31,32]$. This value corresponds to a required photon energy of $9831.57 \mathrm{eV}$.

Besides the lattice constant, we investigated the quality of our diamonds by measuring the rocking curve of each crystal. Theoretically, a peak reflectivity of almost $100 \%$ is predicted for perfect crystals. In contrast, our diamonds show a reflectivity of around $50 \%$. At the same time, the rocking curves are broadened by a factor 1.6 to 2.3 .

\section{EXPERIMENTAL RESULTS}

We set up our x-ray polarimeter at the Nuclear Resonance beamline [33] ID18 of the ESRF, while the storage ring was operating in $7 / 8$ multibunch mode with a maximum ring current of $200 \mathrm{~mA}$ and an electron energy of $6 \mathrm{GeV}$. A scheme of the experimental setup is shown in Fig. 1. The undulator radiation was narrowed in spectral bandwidth to $1.4 \mathrm{eV}$ using 

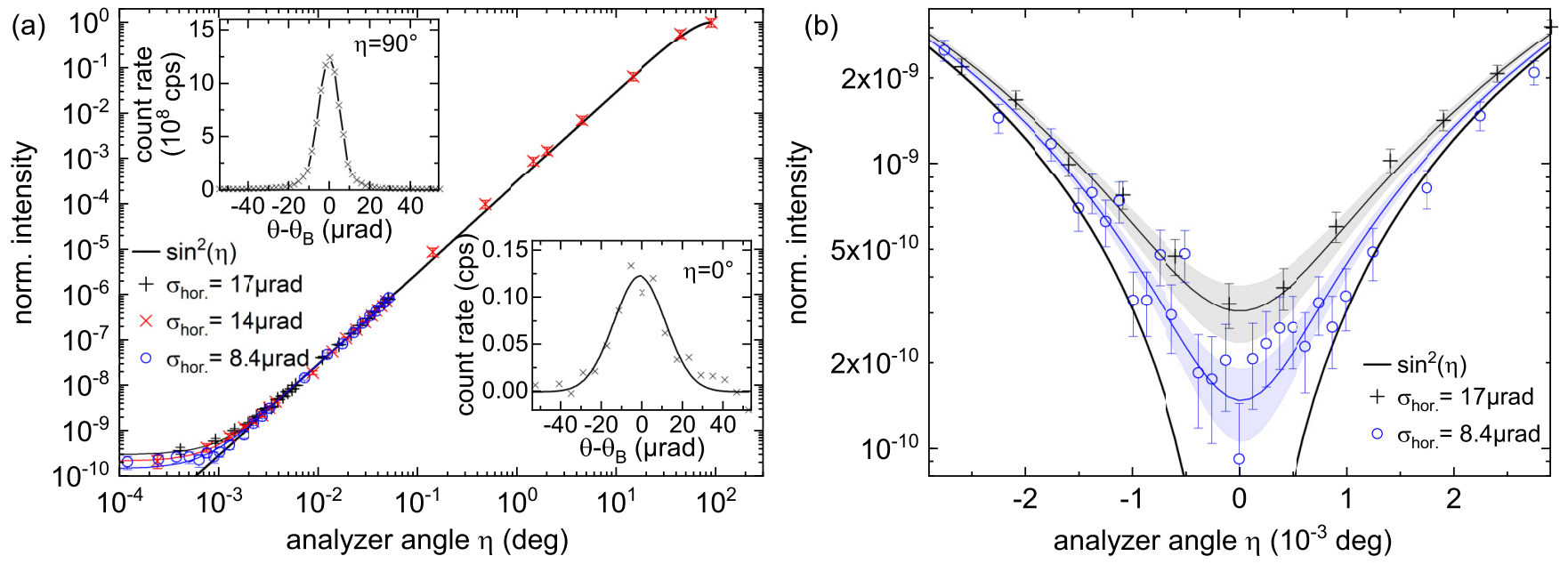

FIG. 3. (a) Intensity behind the polarimeter as a function of the analyzer angle $\eta$ for different horizontal rms beam divergences $\sigma_{\text {hor }}$. The insets show rocking curves of the analyzer in parallel and crossed settings for a horizontal beam divergence $\sigma_{\text {hor }}=8.4 \mu$ rad. The solid lines represent Gaussian fits to the data points, whereas the thick black line is the expected behavior of perfect polarizers. (b) Magnified view close to $\eta=0^{\circ}$. Solid lines are the best parabolic fits to the measured data (symbols) with their $95 \%$ confidence interval as the shaded area. The thick black line represents again the behavior of perfect polarizers.

a silicon 111 high-heat-load monochromator [34]. The photon energy given by the monochromator was calibrated via the $L_{3}$ absorption edge of a 5- $\mu \mathrm{m}$-thick tantalum foil, which has an energy of $9881.1 \mathrm{eV}$ [35]. This allows the choice of correct photon energy within the required $5 \mathrm{eV}$ range. The bandwidth was further reduced by our polarizer to about $0.25 \mathrm{eV}$. Besides the goniometer for the alignment of the incident angle, the analyzer has an additional angular degree of freedom with rotation axes along the beam to vary the analyzer angle $\eta$ from a parallel [Fig. 1(a)] to a crossed setting [Fig. 1(b)]. Depending on $\eta$, we either measured the intensity behind the analyzer with a photodiode (for $\eta>4.5^{\circ}$ ) or a stack of four avalanche photodiodes [36] (for $\eta \leqslant 4.5^{\circ}$ ). Together, both detectors covered a dynamic range of more than 11 orders of magnitude. In addition, we continuously recorded the intensity behind the monochromator and polarizer with ionization chambers in order to monitor the overall stability of the setup.

During the experiment, we varied the beam divergence in the horizontal direction. This was realized by a slit with variable gap and a V-shaped channel cut (VCC). The latter is a silicon crystal with different asymmetries of the inner surfaces. It reduces the divergence by a factor of 3.06 due to asymmetric Bragg diffraction. In order to determine the horizontal divergence $\sigma_{\text {hor }}$, we measured the rocking curve of the analyzer $(\Theta$ scan) in parallel $\left(\eta=90^{\circ}\right)$ and crossed $\left(\eta=0^{\circ}\right)$ settings. In parallel setting, the curve is not broadened by divergence or spectral width of the beam and is therefore a measure for the quality of the crystals. Such a curve is shown in the top inset of Fig. 3(a). Theoretically, this curve should have a full width of half maximum (FWHM) of $8.5 \mu \mathrm{rad}$ for perfect diamond crystals. Due to the imperfections of the diamonds used, we measured a rocking curve FWHM of $12.5 \mu \mathrm{rad}$. In the crossed setting of the polarimeter, the rocking curve of the analyzer is broadened due to the horizontal beam divergence. An example of such a curve is shown in the bottom inset of Fig. 3(a), which has a FWHM of $23.3 \mu \mathrm{rad}$. Here the divergence was already reduced using a VCC. Assuming that these curves are a convolution of the intrinsic reflection curve (the curve in parallel setting) with a Gaussian horizontal divergence, the divergence can be determined by deconvolution. Due to the better signal-to-noise ratio, we did not use the rocking curves measured in the exact crossed setting but close to it. With inserted VCC, we obtained $\sigma_{\text {hor }}=8.4 \mu \mathrm{rad}$. In addition, we performed the same measurement for the pure horizontal beam divergence of the beamline and with an $800-\mu \mathrm{m}$-wide slit about $58 \mathrm{~m}$ far from the source, where we obtained 17 and $14 \mu \mathrm{rad}$, respectively. The vertical divergence $\sigma_{\text {ver }}$ was measured by scanning a $0.1-\mathrm{mm}$-wide slit $32.3 \mathrm{~m}$ far from the source. The corresponding vertical divergence was $6.1 \mu \mathrm{rad}$. Note that these values are rms values of the divergence.

Figure 3(a) shows the intensity measured behind the analyzer as a function of the angle $\eta$ from the parallel to the crossed setting for the three values of $\sigma_{\text {hor }}$. An intensity drop by almost ten orders of magnitude is clearly visible. For comparison, the solid black line shows the behavior of an ideal polarimeter according to $\sin ^{2}(\eta)$. A magnified view close to the crossed setting is shown in Fig. 3(b). For the sake of clarity, only two curves are displayed. The influence of divergence on the purity is obvious. The symbols are measured data points with their statistical uncertainty (square root of counts) as error bars. These data can be approximated by a parabola of the form $a(\eta+b)^{2}+c$ to determine the polarization purity $P=c / a$ as described in Ref. [37]. The fits are represented by colored solid lines with their $95 \%$ confidence interval as the shaded area.

Table I compares the polarization purity $P$ from the fitted data points with the limit of the polarization purity $P_{\text {limit }}$ calculated using Eq. (1). The polarization purity from the fitted data points and the calculated limit agree very well for all three horizontal beam divergences. The maximum error of $P$ given in Table I is the $2 \sigma$ error of the parabolic fit. It is not only due to statistics but also due to long-term drifts of the ACCs. In contrast to a monolithic channel cut, the 
TABLE I. Comparison of the measured polarization purity $P$ and the calculated limit $P_{\text {limit }}$ according to Eq. (1) for three different horizontal rms divergences. The vertical divergence was $6.1 \mu \mathrm{rad}$.

\begin{tabular}{lcc}
\hline \hline$\sigma_{\text {hor }}$ & $P$ & $P_{\text {limit }}$ \\
\hline $17 \mu \mathrm{rad}$ & $(3.0 \pm 0.7) \times 10^{-10}$ & $3.2 \times 10^{-10}$ \\
$14 \mu \mathrm{rad}$ & $(2.2 \pm 0.9) \times 10^{-10}$ & $2.3 \times 10^{-10}$ \\
$8.4 \mu \mathrm{rad}$ & $(1.4 \pm 0.5) \times 10^{-10}$ & $1.1 \times 10^{-10}$ \\
\hline \hline
\end{tabular}

Bragg planes of the crystals do not stay parallel to each other, which results in a loss of intensity. To estimate this effect, we measured the intensity at $\eta=0.6^{\circ}$ before and after each scan. In general, the variation of the throughput was less than $16 \%$ over $1 \mathrm{hr}$ for polarizer and analyzer, respectively.

We also checked the influence of the crystal alignment on the polarization purity at $E_{\mathrm{ph}}=9884 \mathrm{eV}$ and Bragg angle $\theta_{\mathrm{B}}=44.7^{\circ}$, where the polarization component in the plane of diffraction is less suppressed. Contrary to the prediction by Hart and Rodrigues [38], a misalignment of the diamonds to each other degrades the polarization purity. The explanation for this behavior is the lower absorption of diamond as compared to silicon or germanium. Since the parallel polarization component penetrates deeper into the crystal than the perpendicular one, it will interact with more crystal imperfections. Thus, we assume the reflection curve of the parallel component to be broader than the perpendicular one. A misalignment of the crystals will then reduce the overall reflectivity of the latter stronger, which eventually reduces the polarization purity.

\section{CONCLUSION}

In conclusion, we developed an $\mathrm{x}$-ray polarimeter consisting of two compact artificial diamond channel cuts enabling polarization purities limited by the beam divergence. Our work proves the prediction that the polarization purity scales with the square of the rms divergence at this limit [19]. The obtained purity of $1.4 \times 10^{-10}$ exceeds the best value achieved with current silicon-based channel-cut polarimeters. The result was enabled by the precise and stable alignment of eight individual diamond crystals. In the presented ex- periment, we used diamonds produced by chemical vapor deposition, which contain a high number of crystalline defects. These imperfections have no observable influence on the polarization purity. However, they cause a low peak reflectivity of $50 \%-60 \%$ per crystal and thus a low transmittance of polarizer and analyzer. In contrast, polarimetry at photon energies above $10 \mathrm{keV}$ can benefit from imperfections. The broad reflection curves cause a higher integrated reflectivity as well as a higher stability of ACCs. Higher photon energies are, e.g., preferable to enhance the ellipticity induced by vacuum birefringence. At $9.8 \mathrm{keV}$, however, the use of nearly perfect crystals such as high-pressure high-temperature diamonds is indispensable for an optimal performance. High reflectivity is necessary to measure the expected high polarization purity enabled by x-ray sources of the fourth generation. Diffractionlimited storage rings and $\mathrm{x}$-ray free-electron lasers as well as the projected X-ray free-electron laser oscillator sources [39] offer divergences of $1 \mu \mathrm{rad}$ and less and, hence, enable a polarization purity in the order of $10^{-12}$ in the near future. We are therefore confident that even the tiny birefringence induced by a high-power laser into the vacuum will be detected in forthcoming years using polarizers made of diamond. Moreover, there are a lot of applications that benefit from an improved polarization purity as well. Some examples are polarization-resolved imaging [40,41], polarization-resolved spectroscopy [42], quantum optics $[8,10]$, and polarimetry on ultrafast timescales $[11,43]$.

\section{ACKNOWLEDGMENTS}

We acknowledge the European Synchrotron Radiation Facility for the provision of synchrotron radiation facilities and beamtime at the Nuclear Resonance beamline ID18. Furthermore, we would like to thank O. Wehrhan and $\mathrm{H}$. Marschner for characterizing the diamonds, T. Köhler and H. Voigt for designing and constructing the ACCs, R. Bark and D. Hieronymus for developing the ACC photodiode, and J.-P. Celse for technical assistance during the beamtime at ID18. This work has been funded by the Deutsche Forschungsgemeinschaft (DFG) under Grant No. 416700351 within the Research Unit FOR2783/1 and by the Bundesministerium für Bildung und Forschung (BMBF) under Grant No. 05K16SJ2.
[1] C. G. Barkla, Polarisation in Röntgen rays, Nature (London) 69, 463 (1904).

[2] T. S. Toellner, E. E. Alp, W. Sturhahn, T. M. Mooney, X. Zhang, M. Ando, Y. Yoda, and S. Kikuta, Polarizer/analyzer filter for nuclear resonant scattering of synchrotron radiation, Appl. Phys. Lett. 67, 1993 (1995).

[3] D. Siddons, J. Hastings, U. Bergmann, F. Sette, and M. Krisch, Mössbauer spectroscopy using synchrotron radiation: Overcoming detector limitations, Nucl. Instrum. Methods Phys. Res., Sect. B 103, 371 (1995).

[4] R. Röhlsberger, E. Gerdau, R. Rüffer, W. Sturhahn, T. S. Toellner, A. I. Chumakov, and E. E. Alp, X-ray optics for $\mu$ ev-resolved spectroscopy, Nucl. Instrum. Methods Phys. Res., Sect. A 394, 251 (1997).
[5] B. Marx, I. Uschmann, S. Höfer, R. Lötzsch, O. Wehrhan, E. Förster, M. Kaluza, T. Stöhlker, H. Gies, C. Detlefs et al., Determination of high-purity polarization state of x-rays, Opt. Commun. 284, 915 (2011).

[6] B. Marx, K. S. Schulze, I. Uschmann, T. Kämpfer, R. Lötzsch, O. Wehrhan, W. Wagner, C. Detlefs, T. Roth, J. Härtwig, E. Förster, T. Stöhlker, and G. G. Paulus, High-Precision X-Ray Polarimetry, Phys. Rev. Lett. 110, 254801 (2013).

[7] H. Bernhardt, B. Marx-Glowna, K. S. Schulze, B. Grabiger, J. Haber, C. Detlefs, R. Loetzsch, T. Kämpfer, R. Röhlsberger, E. Förster et al., High purity x-ray polarimetry with single-crystal diamonds, Appl. Phys. Lett. 109, 121106 (2016).

[8] K. P. Heeg, H.-C. Wille, K. Schlage, T. Guryeva, D. Schumacher, I. Uschmann, K. S. Schulze, B. Marx, T. Kämpfer, 
G. G. Paulus et al., Vacuum-Assisted Generation and Control of Atomic Coherences at X-Ray Energies, Phys. Rev. Lett. 111, 073601 (2013).

[9] K. P. Heeg, J. Haber, D. Schumacher, L. Bocklage, H.C. Wille, K. S. Schulze, R. Loetzsch, I. Uschmann, G. G. Paulus, R. Rüffer et al., Tunable Subluminal Propagation of Narrow-Band X-Ray Pulses, Phys. Rev. Lett. 114, 203601 (2015).

[10] J. Haber, K. S. Schulze, K. Schlage, R. Loetzsch, L. Bocklage, T. Gurieva, H. Bernhardt, H.-C. Wille, R. Rüffer, I. Uschmann et al., Collective strong coupling of $\mathrm{x}$-rays and nuclei in a nuclear optical lattice, Nat. Photonics 10, 445 (2016).

[11] L. G. Huang, H.-P. Schlenvoigt, H. Takabe, and T. E. Cowan, Ionization and reflux dependence of magnetic instability generation and probing inside laser-irradiated solid thin foils, Phys. Plasmas 24, 103115 (2017).

[12] T. Heinzl, B. Liesfeld, K.-U. Amthor, H. Schwoerer, R. Sauerbrey, and A. Wipf, On the observation of vacuum birefringence, Opt. Commun. 267, 318 (2006).

[13] F. Karbstein, H. Gies, M. Reuter, and M. Zepf, Vacuum birefringence in strong inhomogeneous electromagnetic fields, Phys. Rev. D 92, 071301(R) (2015).

[14] H.-P. Schlenvoigt, T. Heinzl, U. Schramm, T. E. Cowan, and R. Sauerbrey, Detecting vacuum birefringence with $\mathrm{x}$-ray free electron lasers and high-power optical lasers: A feasibility study, Phys. Scr. 91, 023010 (2016).

[15] W. Heisenberg and H. Euler, Folgerungen aus der Diracschen Theorie des Positrons, Z. Phys. 98, 714 (1936).

[16] J. J. Klein and B. P. Nigam, Birefringence of the Vacuum, Phys. Rev. 135, B1279 (1964).

[17] H. Gies, J. Jaeckel, and A. Ringwald, Polarized Light Propagating in a Magnetic Field as a Probe for Millicharged Fermions, Phys. Rev. Lett. 97, 140402 (2006).

[18] R. Cameron, G. Cantatore, A. C. Melissinos, G. Ruoso, Y. Semertzidis, H. J. Halama, D. M. Lazarus, A. G. Prodell, F. Nezrick, C. Rizzo et al., Search for nearly massless, weakly coupled particles by optical techniques, Phys. Rev. D 47, 3707 (1993).

[19] K. S. Schulze, Fundamental limitations of the polarization purity of x rays, APL Photonics 3, 126106 (2018).

[20] H. Cole, F. W. Chambers, and C. G. Wood, X-ray polarizer, J. Appl. Phys. 32, 1942 (1961).

[21] M. Hart, X-ray polarization phenomena, Philos. Mag. B 38, 41 (1978).

[22] M. Hart, D. Siddons, Y. Amemiya, and V. Stojanoff, Tunable $\mathrm{X}$-ray polarimeters for synchrotron radiation sources, Rev. Sci. Instrum. 62, 2540 (1991).

[23] M. Renninger, Umweganregung, eine bisher unbeachtete Wechselwirkungserscheinung bei Raumgitterinterferenzen, Z. Phys. 106, 141 (1937).

[24] J. Z. Tischler and B. W. Batterman, Determination of phase using multiple-beam effects, Acta Crystallogr., Sec. A: Found. Crystallogr. 42, 510 (1986).

[25] Y. Shvyd'ko, S. Stoupin, V. Blank, and S. Terentyev, Near$100 \%$ Bragg reflectivity of $\mathrm{x}$-rays, Nat. Photonics 5, 539 (2011).
[26] H. Holloway, K. C. Hass, M. A. Tamor, T. R. Anthony, and W. F. Banholzer, Isotopic dependence of the lattice constant of diamond, Phys. Rev. B 44, 7123 (1991).

[27] T. Yamanaka, S. Morimoto, and H. Kanda, Influence of the isotope ratio on the lattice constant of diamond, Phys. Rev. B 49, 9341 (1994).

[28] K. Haruna, H. Maeta, K. Ohashi, and T. Koike, Thermal expansion coefficient of synthetic diamond single crystal at low temperatures, Jpn. J. Appl. Phys. 31, 2527 (1992).

[29] T. Saotome, K. Ohashi, T. Sato, H. Maeta, K. Haruna, and F. Ono, Thermal expansion of a boron-doped diamond single crystal at low temperatures, J. Phys.: Condens. Matter 10, 1267 (1998).

[30] T. Sato, K. Ohashi, T. Sudoh, K. Haruna, and H. Maeta, Thermal expansion of a high purity synthetic diamond single crystal at low temperatures, Phys. Rev. B 65, 092102 (2002).

[31] W. L. Bond, Precision lattice constant determination, Acta Crystallogr. 13, 814 (1960).

[32] We calculated from the $a$ of our CVD diamonds and the $a$ given by Sato et al. [30] that $\Delta a / a$ is due to dislocation in the range of $4.8 \times 10^{-5}$.

[33] R. Rüffer and A. I. Chumakov, Nuclear resonance beamline at ESRF, Hyperfine Interact. 97, 589 (1996).

[34] A. I. Chumakov, I. Sergeev, J.-P. Celse, R. Rüffer, M. Lesourd, L. Zhang, and M. Sánchez del Río, Performance of a silicon monochromator under high heat load, J. Synchrotron Radiat. 21, 315 (2014).

[35] J. A. Bearden and A. F. Burr, Reevaluation of x-ray atomic energy levels, Rev. Mod. Phys. 39, 125 (1967).

[36] A. Q. Baron, Detectors for nuclear resonant scattering experiments, Hyperfine Interact. 125, 29 (2000).

[37] K. S. Schulze, B. Marx, I. Uschmann, E. Förster, T. Stöhlker, and G. G. Paulus, Determination of the polarization state of $x$ rays with the help of anomalous transmission, Appl. Phys. Lett. 104, 151110 (2014).

[38] M. Hart and A. R. D. Rodrigues, Tuneable polarizers for x-rays and neutrons, Philos. Mag. B 40, 149 (1979).

[39] B. Adams, G. Aeppli, T. Allison, A. Q. R. Baron, P. Bucksbaum, A. I. Chumakov, C. Corder, S. P. Cramer, S. DeBeer, Y. Ding et al., Scientific opportunities with an x-ray free-electron laser oscillator, arXiv:1903.09317.

[40] K. Hirano, X-ray polarization-contrast imaging of crystals, J. Appl. Phys. 79, 3365 (1996).

[41] B. A. Palmer, G. R. Edwards-Gau, B. M. Kariuki, K. D. Harris, I. P. Dolbnya, and S. P. Collins, X-ray birefringence imaging, Science 344, 1013 (2014).

[42] A. T. Schmitt, Y. Joly, K. S. Schulze, B. Marx-Glowna, I. Uschmann, B. Grabiger, H. Bernhardt, R. Loetzsch, A. Juhin, H.-C. Wille, H. Yavaş, G. G. Paulus, and R. Röhlsberger, Disentangling $\mathrm{X}$-ray dichroism and birefringence in high-purity polarimetry, arXiv:2003.00849.

[43] T. Wang, T. Toncian, M. S. Wei, and A. V. Arefiev, Structured targets for detection of megatesla-level magnetic fields through Faraday rotation of XFEL beams, Phys. Plasmas 26, 013105 (2019). 